\title{
Reganancia de peso después de la cirugía bariátrica*
}

\author{
Dra. KARIN PAPAPIETRO V. ${ }^{1}$
}

1 Unidad de Nutrición, Departamento de Cirugía, Hospital Clínico de la Universidad de Chile. Santiago, Chile.

\begin{abstract}
Weight gain after bariatric surgery

Among obese subjects, bariatric surgery is very effective to achieve weight loss, to improve metabolic disturbances such as diabetes mellitus and to improve quality of live. However, long term follow up studies show that an important proportion of patients gain weight again. This phenomenon commonly occurs between the third and sixth postoperative year. The main risk factors for weight gain are behavioral and psychological issues, that can be detected in the preoperative assessment. This reinforces the concept that bariatric surgery must be managed by experienced multidisciplinary teams
\end{abstract}

Key words: Obesity, bariatric surgery, weight gain.

\section{Resumen}

La cirugía para la obesidad ha probado ser el tratamiento más efectivo para reducir el exceso de peso de cualquier magnitud, lo que favorece la mejoría de las comorbilidades metabólicas, especialmente de la diabetes tipo 2 y de la calidad de vida. Los estudios con seguimiento de largo plazo muestran que un porcentaje importante de pacientes operados regana peso, lo que se observa más frecuentemente entre el tercer y sexto año postoperatorio. Existen cambios anatómicos y factores dependientes de la técnica quirúrgica que pueden explicar la reganancia de peso, pero son los aspectos conductuales y sicológicos, los principales factores de riesgo para que un paciente que ha reducido exitosamente su exceso de peso vuelva a engordar. Varios de estos aspectos, son detectables en la etapa preoperatoria, lo que confirma que la cirugía bariátrica debe ser realizada en centros de excelencia que cuenten con equipo multidisciplinario.

Palabras clave: Cirugía bariátrica, resultados, reganancia de peso.

La cirugía bariátrica ha demostrado ser eficaz para el tratamiento de la obesidad mórbida y las comorbilidades asociadas. Los estudios han demostrado que el tratamiento quirúrgico logra una mayor pérdida de peso que el tratamiento médico para la obesidad de cualquier grado de severidad ${ }^{1}$. Sin embargo, con ningún tipo de cirugía se ha logrado asegurar la mantención del peso en el largo plazo.

\section{Reducción del peso}

En los estudios de revisión, se observan cifras de reducción del exceso de peso con un amplio rango. Entre 29 y $87 \%$ con banding gástrico (BG), la cirugía más común en el mundo. Las técnicas que incorporan un componente de malabsorción como el bypass gástrico (BPG) y la diversión bi-

\footnotetext{
* Recibido el 14 de octubre de 2011 y aceptado para publicación el 10 de noviembre de 2011.

Correspondencia: Dra. Karin Papapietro V.

Santos Dumont 999, Santiago, Chile.

kpapapie@gmail.com
} 
liopancreática (DBP) reducen entre 48 y $85 \%$ del exceso de peso. Estas técnicas, también producen una rápida llegada de alimentos al intestino distal, donde existe gran concentración de células L que producen enteropeptidos llamados incretinas ( PYY, GIP,GLP-1). Estas entero hormonas tienen efecto sobre la reducción del apetito, la motilidad intestinal, la resistencia insulínica y el control de la glicemia, por lo que también se las relaciona con la rápida mejoría de las comorbilidades metabólicas observadas antes que ocurra una disminución significativa del peso. La gastrectomía subtotal vertical (GSTV) o manga gástrica, inicialmente propuesta para superobesidad, reporta reducción entre 33 y $66 \%$ del exceso de peso. Esta técnica restrictiva, debido a la gran resección gástrica, reduce drásticamente los niveles de la hormona orexigena grhelina y también estimula la producción de incretinas por causa del acelerado vaciamiento del tubo gástrico remanente ${ }^{2}$.

Con todas las técnicas, la reducción del peso ocurre durante los 12 a 18 meses que siguen a la cirugía, aun en pacientes con superobesidad. La principal causa de la pérdida de peso es la obvia disminución de la ingesta calórica, debido a la restricción alimentaria impuesta por la cirugía. Además se produce anorexia y estímulo de la lipolisis por cambios en los niveles de hormonas que controlan el apetito y el metabolismo energético. A lo anterior se suma la malabsorción parcial cuando se utilizan técnicas que incluyen bypass de segmentos intestinales y aumento en la velocidad de tránsito.

Se ha estudiado si existen aspectos de la técnica quirúrgica que puedan influir en la reducción del peso. En BPG, la reducción del diámetro de la anastomosis gastroyeyunal no ha demostrado influir en la cantidad de peso reducido ${ }^{3}$.

Por otra parte, el largo del asa alimentaria tampoco tiene efecto sobre la pérdida de peso según una revisión recientemente publicada ${ }^{4}$. Sólo en pacientes superobesos, un estudio reportó mayor pérdida de peso en pacientes con asa alimentaria de $150 \mathrm{~cm}$ que en pacientes con un asa de $70 \mathrm{cms}$. Estos resultados, sin embargo, no se han reproducido en pacientes con obesidad de menor IMC $\mathrm{IM}^{5}$. Por tanto, en pacientes con obesidad mórbida, el largo del asa alimentaria no influye en la reducción y tampoco en la mantención del peso a largo plazo $^{3-5}$.

En manga gástrica, Weiner realizó en 117 pacientes 3 tamaños del tubo gástrico, un grupo calibrado con bujía 32Fr otro con bujía 44Fr y un tercer grupo sin calibración. Evaluó la evolución del IMC durante 5 años y sus resultados mostraron que los pacientes que quedaron con menos estómago remanente y en los que se usó una bujía menor, tuvieron una pérdida de peso significativamente mayor que los pacientes en que se resecó menos estómago y no se utilizó bujía para calibrar ${ }^{6}$. Al parecer el tamaño del tubo gástrico es el único factor de la técnica quirúrgica que influye en la pérdida de peso.

Además de la técnica quirúrgica, en el análisis de la reducción de peso, se ha considerado aspectos conductuales. Leite y cols ${ }^{7}$, evaluaron la influencia de la composición de la dieta postoperatoria en el proceso de descenso de peso. Se calculó el promedio de baja de peso mensual, se analizó la frecuencia de consumo de alimentos y la proporción de los diferentes macronutrientes (lípidos, proteínas y carbohidratos) que los pacientes incluían en su dieta después del BPG. Sus resultados mostraron que los pacientes que comían más veces en el día reducían menos peso. Por otra parte, también aquellos que preferían carbohidratos y comidas con elevada carga glicémica incorporando chocolate, harinas, arroz y papas, redujeron significativamente menos peso comparado con los que elegían alimentos más proteicos y con menor indice glicémico. Mediante una fórmula matemática, ellos estimaron que las comidas con alta carga glicémica dieron cuenta del $63 \%$ del promedio mensual de reducción del peso después de un bypass gástrico y que por cada 10 puntos menos de carga glicémica en la dieta, se lograba una mejoría del $2 \%$ en la reducción mensual de peso. Los autores recomendaron que después del BPG la frecuencia de comida y la composición de la dieta debía ser asesorada para optimizar la reducción del peso. Sugerman, propuso que el BPG era una mejor alternativa quirúrgica que la gastroplastía para comedores de dulce, reconociendo la negativa influencia del consumo de dulces en el resultado de la cirugía bariátrica ${ }^{8}$.

\section{Reganancia de peso}

El aumento de peso posterior a la cirugía bariátrica es reconocido en todas las series con seguimiento mayor a 2 años. Se ha reportado desde 20 a $87 \%$ de pacientes con reganancia de peso en diversas series $^{2,4,9}$. La magnitud de la ganancia de peso descrita, es en promedio $10 \mathrm{~K}$ con rango de $0,5 \mathrm{~K}$ y hasta $60 \mathrm{~K}$. El aumento de peso ocurre más frecuentemente entre 3 y 6 años después de la cirugía. La reganancia de un $25 \%$ del peso perdido previamente por efecto de la cirugía bariátrica ha sido considerado por algunos autores como un criterio suficiente para realizar cirugía revisional. En otros estudios se ha considerado que la reganancia de peso ha favorecido la reaparición de comorbilidades como la Diabetes.

Se han reportado diversos factores que influyen en el aumento de peso posterior a una cirugía exitosa. Una observación frecuente, es que los pacientes que lograron menos reducción de peso con la operación tienden a una rápida reganancia. 
Según tipo de operación, el banding gástrico aparece con mayor reganancia de peso según un estudio con seguimiento a 10 años. Mantener una pérdida de peso de al menos $20 \%$ se observa sólo en $28 \%$ de los pacientes con banding gástrico comparado con $74 \%$ de los pacientes con $\mathrm{BPG}^{10}$. En cuanto aspectos de la técnica quirúrgica, Abbu Dayyieh y cols ${ }^{11}$, analizaron con un modelo de regresión, la relación entre diámetro de la anastomosis gastroyeyunal y el cambio de peso en 167 pacientes con 4 años de evolución postoperatoria. En esta serie, el $59 \%$ de los pacientes había reganado más de $20 \%$ del peso perdido previamente. Los autores estimaron que el aumento del diámetro de la anastomosis tenía valor predictivo para la reganancia de peso, su hipótesis es que el aumento en el diámetro de la anastomosis provocaría pérdida de la contención y rápido vaciamiento del pouch gástrico con disminución en la saciedad postprandial facilitando la mayor ingesta de alimentos. Christou, en pacientes con obesidad mórbida y superobesos que tenían más de 10 años de BPG, comparó la evolución del peso según el largo del asa de la Y de Roux. Los autores concluyeron que el largo del asa no influye en la reganancia de peso a largo plazo ${ }^{12}$.

En el seguimiento de pacientes con manga gástrica, Braghetto y cols, ha descrito que con el paso del tiempo existe importante aumento de la capacidad de la manga, evaluando con técnicas radiológicas e isotópicas. Al comparar imágenes postoperatorias precoces de la capacidad de la manga calibrada con bujías 32, con imágenes del mismo paciente tomadas años después, se ha encontrado una capacidad del tubo gástrico significativamente mayor ${ }^{13}$.

Los aspectos hormonales también han sido considerados. En un estudio en animales con modelo experimental de bypass gástrico, se evaluó niveles de varias incretinas y se observó que sólo los niveles de PYY mostraron relación con reganancia de peso. Los autores, a partir de sus resultados, plantearon como hipótesis que existirían pacientes con imposibilidad de mantener elevados los niveles de PYY (hormona anorexigena y que estimula la lipolisis), lo que produciría aumento en el deseo de ingerir alimentos con la consecuente ganancia de peso ${ }^{14}$.

Después de la reducción de peso provocada por la cirugía, se produce una baja en el metabolismo basal, lo que contribuye a la reganancia de peso en el largo plazo. Esto es concordante con los resultados de estudios que muestran que el sedentarismo en pacientes con antecedente de BPG, es una variable predictora de reganancia de peso. La disminución en la tasa metabólica puede ser contrarrestada con ejercicio físico, resaltando la importancia de incorporar actividad física para evitar la reganancia de peso ${ }^{15,16}$.

Los aspectos de la conducta del paciente son los factores más relacionados con la reganacia de peso después de cualquier tipo de tratamiento para la obesidad.

El aumento progresivo en la ingesta calórica en pacientes con cirugía bariátrica es bien conocido. En un estudio de Brolin $^{17}$, se reportó que los pacientes 6 meses después de un BPG ingerían aproximadamente $900 \mathrm{Cal} /$ día y después de 2,5 años aumentaban a $1.400 \mathrm{Cal} /$ día. Aunque esta cantidad no justificaría un aumento de peso, hay que recordar que los pacientes pueden subestimar su ingesta real hasta en un $40 \%$. Por otra parte, este incremento del consumo calórico produce mayor efecto lipogénico en pacientes con bajo metabolismo.

El consumo frecuente de carbohidratos se asocia a una mayor ganancia de peso $^{7}$. Independiente de su aporte calórico, los azúcares y alimentos de mayor índice glicémico estimulan la producción de insulina y disminuyen la lipolisis.

En un análisis de los factores conductuales que facilitan la reganancia después de la cirugía, encontraron que el comer por impulso aumentaba en 5 veces la probabilidad de ganar peso, al igual que el no asistir a controles periódicamente. Además los individuos con malestar sicológico tenían 25 veces más posibilidad de reganar peso ${ }^{18}$. Estas estimaciones son concordantes con los estudios que muestran que pacientes que después de la cirugía bariátrica padecen enfermedades psiquiátricas, tienen alta tasa de reganancia de peso. Rutledge ${ }^{19}$, demostró una correlacion positiva entre el número de enfermedades psiquiátricas diagnosticadas después de la cirugía y la magnitud del aumento de peso.

Todas las técnicas de cirugía bariátrica permiten reducir exitosamente el exceso de peso, aun en los casos de obesidad más severa. Sin embargo, ninguna operación asegura la mantención del peso reducido. En la reganancia de peso participan cambios adaptativos como el aumento en la capacidad del estómago remanente, aumento del diámetro de las anastómosis y la adaptación intestinal posterior a una enterectomía. Todos son factores anatómico-funcionales que facilitan y potencian el aumento de la ingestión de alimentos. Pero, el factor mas relevante en el aumento de peso después de la cirugía bariátrica, es la pérdida del control de la alimentación retornando a una conducta alimentaria inapropiada cuyas características más frecuentes son: El desorden en los horarios de comida con incorporación frecuente de snacks (picoteos), la ingestión de alimentos por ansiedad o en relación a estímulos emocionales y la frecuente elección de alimentos con alta carga glicémica. La mayoría de estas conductas se favorecen en pacientes con malestar sicológico. Los trastornos ansiosos motivados por eventos de estrés como duelos, divorcios o pérdida del trabajo, los trastornos de 
personalidad o enfermedades siquiátricas como la depresión o el alcoholismo, son una causa frecuente de pérdida del control y del autocuidado que experimentan los pacientes que reganan peso. Además del alejamiento de los controles periódicos necesarios con los profesionales encargados del cuidado del paciente con cirugía bariátrica ${ }^{18-22}$.

En conclusión, los factores no quirúrgicos, los sicológicos y conductuales son más relevantes que los factores quirúrgicos en la reganancia de peso del paciente con cirugía bariátrica, porque los cambios anatómicos postoperatorios también tienen relación con la conducta alimentaria del paciente y sobretodo porque hay pacientes que mantienen el peso reducido a pesar de tener cambios anatómicos.

Muchos de los factores conductuales y sicológicos estudiados, se pueden detectar o prever en la etapa preoperatoria, en la cual se puede planificar e iniciar un tratamiento, pero lo más importante, es informar al paciente cuales son sus factores de riesgo para reganancia de peso que no serán modificados por la cirugía. Esto permite esclarecer que la responsabilidad de la mantención del peso después de la cirugía es responsabilidad del paciente y no de la técnica quirúrgica o del cirujano. Los factores involucrados en la reganancia de peso, confirman que el tratamiento quirúrgico de la obesidad debe ser realizado en centros de excelencia, con cirujanos y equipo multidisciplinario expertos, capaces de enfrentar todos los aspectos del paciente candidato a cirugía bariátrica.

\section{Referencias}

1. Csendes A, Burdiles P, Papapietro K, Burgos AM. Comparación del tratamiento médico y quirúrgico en pacientes con obesidad grado III (obesidad mórbida). Rev Med Chile 2009;137:559-66.

2. Mechanick JI, Kushner RF, Sugerman HJ, GonzálezCampoy JM, Collazo-Clavell ML, Spitz AF, et al. American Association of Clinical Endocrinologists, The Obesity Society, and American Society for Metabolic \& Bariatric Surgery Medical Guidelines for Clinical Practice for the Perioperative Nutritional, Metabolic, and Nonsurgical Support of the Bariatric Surgery Patient. Surgery for Obesity and Related Diseases 2008;S10984.

3. Brolin RE. La Marca LB, Kenler HA . Cody RP. Malabsorptive Gastric Bypass in Patients With Superobesity J Gastrintes Surg. 2002;6:195-205.

4. Brolin RE. Weight gain after short- and long-limb gastric bypass in patients followed for longer than 10 years. Ann Surg. 2007;246:163-4.

5. Stefanidis D, Kuwada TS, Gersin KS. The importance of the length of the limbs for gastric bypass patients an evidence-based review. Obes Surg. 2011;211:119-24.

6. Weiner RA, Weiner S, Pomhoff I, Jaciobi C, Wakarewics W, Weigand G. Laparoscopic sleeve gastrectomy-influence of sleeve size and resected gastric volume. Obes Surg. 2007;17:1297-305.

7. Leite Faria S, Pereira Faria O, Carvalho Lopes T, Vieira Galvão M, Oliveira Kelly E, Kiyomi Ito M. Relation Between Carbohydrate Intake and Weight Loss After Bariatric Surgery. Obes Surg. 2009;19:708-16.

8. Sugerman HJ, Starkey J, Birkenhauer R. A randomized prospective trial of gastric bypass versus vertical banded gastroplasty for morbid obesity and their effects on sweets versus non-sweets eaters. Ann Surg. 1987;205:613-24.

9. Kofman, Lent M, Swencionis Ch. Maladaptive Eating Patterns, Quality of Life, and Weight Outcomes Following Gastric Bypass: Results of an Internet Survey Michele D. Obesity 2010;18:1938-43.

10. Sjöström L, Lindroos AK, Peltonen M, Torgerson J, Bouchard C, Björn C, et al (Swedish Obese Subjects Study Scientific Group). Lifestyle, diabetes, and cardiovascular risk factors 10 years after bariatric surgery. N Engl J Med. 2004;351:2683-93.

11. Dayyeh AB, Lautz DB, Thompson Ch. Gastrojejunal Stoma Diameter Predicts Weight Regain After Rouxen-Y Gastric Bypass. Clin Gastroenterol Hepatol. 2011;9:228-33.

12. Christou NV, Look D, Maclean L. Weight gain after short and long- limb gastric bypass in patients followed for longer than 10 years. Ann Surg. 2006;244:734-40.

13. Braghetto I, Cortes C, Herquiñigo D, Csendes P, Rojas A, Mushle M, et al. Evaluation of the Radiological Gastric Capacity and Evolution of the BMI 2-3 Years After Sleeve Gastrectomy. Obes Surg. 2009;19:1262-9.

14. Meguid M, Glade MJ, Middleton FA. Weight regain after Roux-en-Y: A significant 20\% complication related to PYY. Nutrition 2008;24:832-42.

15. MacLean PS, Higgins JA, Johnson GC, Fleming-Elder BK, Donahoo TD, Melanson EL, et al. Enhanced metabolic efficiency contributes to weight regain after weight loss in obesity-prone rats. Am J Physiol Regul Integr Comp Physiol. 2004:287:1306-15.

16. Leite Faria S, Kelly E, Faria OP. Energy Expenditure and Weight Regain in Patients Submitted to Roux-en-Y Gastric Bypass. Obes Surg. 2009;19:856-9.

17. Brolin RE, Robertson LB, Kenler HA, Cody RP. Weight loss and dietary intake after vertical banded gastroplasty and Roux-en-Y gastric bypass. Ann Surg. 1994;220:782-90.

18. PatakyZ, Carrard I, Golay A. Psychological factors and weight loss in bariatric surgery. Curr Opin Gastroenterol. 2011;27:167-73.

19. Rutledge T, Groesz LM, Savu M. Psychiatric Factors and Weight Loss Patterns Following Gastric Bypass Surgery in a Veteran Population. Obes Surg. 2011;21:29-35. 
20. Leite Faria S, de Oliveira Kelly E, Pereira Faria O, Kiyomi Ito M. Snack-eating patients experience lesser weight loss after Roux-en-Y gastric bypass surgery. Obes Surg. 2009;19:1293-6.

21. Odom J, Zalesin KC, Washington TL, Miller WW, Hakmeh B, Zaremba DL, et al. Behavioral predictors of weight regain after bariatric surgery. Obes Surg. 2010;20:349-56.

22. Sarwer B, Dilks RJ, West-Smith L. Dietary intake and eating behavior after bariatric surgery: threats to weight loss maintenance and strategies for success. Surg Obes Rel Dis. 2011;7:644-51. 